\title{
Quartic Non-polynomial Spline Method for Singularly Perturbed Differential- difference Equation with Two Parameters
}

\author{
Gemadi Roba Kusi, Tesfaye Aga Bullo*, and Gemechis File Duressa \\ Department of Mathematics, Jimma University, Jimma, P.O. Box 378, Ethiopia
}

Received: March 15, 2021, Revised: April 27, 2021, Accepted: April 29, 2021, Available Online: May 03, 2021

\begin{abstract}
Quartic non-polynomial spline method is presented to solve the singularly perturbed differential-difference equation containing two parameters. The considered equation is transformed into an asymptotical equivalent differential equation, and the derivatives are replaced finite difference approximation using the quartic non-polynomial spline method. The convergence analysis of the method has been established. Numerical experimentation is carried out on model examples, and the results are presented both in tables and graphs. Furthermore, the present method gives a more accurate solution than some existing methods reported in the literature.
\end{abstract}

Keywords: Quartic non-polynomial, differential-difference, two-parameters, accurate solution.

This work is licensed under a Creative Commons Attribution-Non Commercial 4.0 International License.

\section{Introduction}

Numerical analysis is both a science and an art. As a science, it is concerned with the processes by which mathematical problems can be solved by appropriate methods. While, as an art numerical analysis is concerned with choosing that procedure that is suitable for the solution of a particular problem. Numerical answers to a problem generally cover errors that arise in inherent in the mathematical formulation of the problem or approximate to the physical situation and suffered in finding the solution numerically. If the small positive constant number multiples with the highest-order derivative of a given differential equation, then the obtained equation is known as a singularly perturbed differential equation. The small positive parameter is known as the perturbation parameter. In singular perturbation theory, the study of differential equations which are modified by the addition of small coefficients multiplying the higher-order derivative is of importance in many fields, [1]-[5].

Singular perturbation problems are the differential or difference equations that arise as a result of the modeling of reallife phenomena and whose solutions exhibit the boundary layer. Based on the parameters, the perturbation and/or delay parameters they involved, singularly perturbed problems can be categorized into the singularly perturbed differential equations or singularly perturbed differential-difference equations. Many researchers, like in, [7]-[16] have been providing different numerical methods for solving singularly perturbed differentialdifference equations. But, most of those author's considered the stated problem when it involves one perturbation parameter. Few scholars like in [9],[14],[17] have been developed numerical schemes to solve singularly perturbed problems with two parameters. For the problems that contain two perturbation parameters and involve delay term in the convection term proposed by Sahu and Mohapatra, [9] who tried to develop a parameter uniform numerical method.

However, this developed method and most of the classical methods produce good results only when the perturbation parameter in the convection term $\mu$ is much less than the perturbation parameter in the diffusion coefficient $\delta$, (i.e., $\mu<<\varepsilon)$. This difficulty is caused due to $\mu$ in convection term which implies the existence of the two boundary layers in the solution. Moreover, classical numerical methods give good accurate solutions only when the step length the solution domain $h<\varepsilon$. This leads to huge systems of equations which is costly to solve. Thus, in this paper, we present a quartic non-polynomial spline method that produces a more accurate solution for singularly perturbed differential-difference equations involving two parameters when $\varepsilon \leq \mu \leq h$.

\section{Description of the Numerical Method}

We consider the singularly perturbed differential-difference equation with two parameters of the form:

$$
\begin{aligned}
& \varepsilon y^{\prime \prime}(x)+\mu a(x) y^{\prime}(x-\delta)-b(x) y(x)=g(x), \\
& x \in \Omega=(0,1)
\end{aligned}
$$

with the interval and boundary condition:

$$
y(x)=\phi(x),-\delta \leq x \leq 0, y(1)=\beta \text {. }
$$

where $0<\varepsilon, \mu<<1$ are the perturbation parameters. The delay parameter is $\delta$, and satisfies $\delta<\varepsilon$. Functions $a(x), b(x), g(x)$ and $\phi(x)$ are continuous on $\Omega$, and the constant number $\beta$ is given. Furthermore, assume that $a(x) \geq a>0$ and $b(x) \geq b>0, \forall x \in \bar{\Omega}$.

By Taylor's series expansion as:

$$
y^{\prime}(x-\delta)=y^{\prime}(x)-\delta y^{\prime \prime}(x)+O\left(\delta^{2}\right)
$$

Substituting Eq. (3) into Eq. (1), gives the asymptotical equivalent boundary value problem:

$$
\varepsilon y^{\prime \prime}(x)+\mu p(x) y^{\prime}(x)-q(x) y(x)=f(x)
$$

with the boundary conditions, 
$y(0)=\phi_{0}=\phi\left(x_{0}\right), \quad y(1)=A$

where: $\quad p(x)=\frac{\varepsilon a(x)}{\varepsilon-\delta \mu a(x)}, q(x)=\frac{\varepsilon b(x)}{\varepsilon-\delta \mu a(x)}$, and $f(x)=\frac{\varepsilon g(x)}{\varepsilon-\delta \mu a(x)}$.

Consider for $0<p_{0} \leq p(x) \leq p_{1}$ and $q(x) \geq q \geq 0$, the homogenous part of Eq. (4) is written as;

$$
\varepsilon y^{\prime \prime}(x)+\mu p_{1} y^{\prime}(x)-q y(x)=0
$$

The characteristic equation of Eq. (6) is $\varepsilon m^{2}+\mu p_{1} m-q=0$, and assume it has two real solution

$m_{1,2}=\frac{-\mu p_{1} \pm \sqrt{\left(\mu p_{1}\right)^{2}+4 \varepsilon q}}{2 \varepsilon}$.

The situation of the layer is characterized by the case, for $\varepsilon<<\mu$, as $\mu \rightarrow 0$, and $q \geq 0$ which suggests that $m_{1} \approx \frac{-\mu p_{1}}{\varepsilon}$ and $m_{2} \approx 0$. Hence, in this case for $\varepsilon<<\mu$, the complementary solution to Eq. (6) is

$$
y(x)=C_{1}+C_{2} e^{\frac{-\mu p_{1}}{\varepsilon} x},
$$

where $C_{1}$ and $C_{2}$ are arbitrary constants.

Let $N$ be a positive integer and a uniform mesh $\Delta$ with nodal point $x_{i}$ on $[0,1]$ such that: $\Delta: 0=x_{0}<x_{1}<x_{2}<\ldots<x_{N-1}<x_{N}=1$, $x_{i}=i h, i=0,1,2, \ldots, N$; where $h=\frac{1}{N}$, and $N$ is the number of intervals. For each segment $\left[x_{i}, x_{i+1}\right], i=1,2, \ldots, N-1$, let us the non-polynomial quartic spline $S_{\Delta}(x)$ defined by:

$$
\begin{aligned}
& S_{\Delta}(x)=a_{i} \sin k\left(x-x_{i}\right)+b_{i} \cos k(x- \\
& \left.x_{i}\right)+c_{i}\left(x-x_{i}\right)^{2}+d_{i}\left(x-x_{i}\right)+e_{i},
\end{aligned}
$$

where $a_{i}, b_{i}, c_{i}, d_{i}$ and $e_{i}$ are real finite constants to be determined and $k$ is an arbitrary parameter that will be used to increase the accuracy of the method. Denoting $y_{i}$ be an approximation to $y\left(x_{i}\right)$ obtained by the segments $S_{\Delta}(x)$ of the spline function passing through the points $\left(x_{i}, S_{\Delta}\right)$ and $\left(x_{i+1}, S_{\Delta+1}\right)$. To determine the coefficients of Eq. (8), we do not only require that $S_{\Delta}(x)$ satisfies interpolator conditions at $x_{i}$ and $x_{i+1}$ but also continuity condition of the first, and the third derivatives at the nodes $\left(x_{i}, S_{\Delta}\right)$ and $\left(x_{i+1}, S_{\Delta+1}\right)$. Symbolizing:

$$
\begin{aligned}
& S_{\Delta}\left(x_{i}\right)=y_{i}, \quad S_{\Delta}\left(x_{i+1}\right)=y_{i+1} \\
& S_{\Delta}^{\prime \prime}\left(x_{i}\right)=M_{i}, \quad S_{\Delta}^{\prime \prime}\left(x_{i+1}\right)=M_{i+1} \\
& S^{(4)}\left(x_{i}\right)=\frac{1}{2}\left(F_{i}+F_{i+1}\right)
\end{aligned}
$$

We get through a long straightforward calculation,

$a_{i}=\frac{1}{k^{2} \sin \theta}\left(M_{i}-M_{i+1}\right)+\frac{1-\cos \theta}{2 k^{4} \sin \theta}\left(F_{i}+F_{i+1}\right)$,

$b_{i}=\frac{F_{i}+F_{i+1}}{2 k^{4}}$,

$c_{i}=\frac{M_{i}}{2}+\frac{F_{i}+F_{i+1}}{4 k^{2}}$,

$d_{i}=\frac{1}{h}\left(y_{i+1}-y_{i}\right)-\left(\frac{1}{h k^{2}}+\frac{h}{2}\right) M_{i}+\frac{1}{h k^{2}} M_{i+1}-\frac{h}{4 k^{2}}\left(F_{i}+F_{i+1}\right)$,

$e_{i}=y_{i}-\frac{1}{2 k^{4}}\left(F_{i}+F_{i+1}\right)$.

Expending the continuity condition of the first derivatives at knots, $S_{\Delta-1}^{\prime}\left(x_{i}\right)=S_{\Delta}^{\prime}\left(x_{i}\right)$, leads to:

$$
\begin{aligned}
& \frac{4 h k^{3} \sin \theta}{2(1-\cos \theta)-h k \sin \theta}\left[\frac{y_{i-1}-2 y_{i}+y_{i+1}}{h^{2}}\right]+ \\
&\left(F_{i-1}+F_{i+1}\right)+2 F_{i}= \frac{2 k\left(2 h k \cos \theta+h^{2} k^{2} \sin \theta-2 \sin \theta\right)}{h[2(1-\cos \theta)-h k \sin \theta]} M_{i-1}+ \\
& \frac{2 k\left(4 \sin \theta+h^{2} k^{2} \sin \theta-2 h k(\cos \theta+1)\right)}{h[2(1-\cos \theta)-h k \sin \theta]} M_{i}+ \\
& \frac{4 k(h k-\sin \theta)}{h[2(1-\cos \theta)-h k \sin \theta]} M_{i+1} .
\end{aligned}
$$

Likewise, using the continuity of the third derivatives at knots, $S_{\Delta-1}^{\prime \prime \prime}\left(x_{i}\right)=S_{\Delta}^{\prime \prime \prime}\left(x_{i}\right)$, we get:

$$
\left(F_{i-1}+F_{i+1}\right)+2 F_{i}=\frac{2 k^{2} \cos \theta}{1-\cos \theta} M_{i-1}-\frac{2 k^{2}(\cos \theta+1)}{1-\cos \theta} M_{i}+\frac{2 k^{2}}{1-\cos \theta} M_{i+1}
$$

By substituting Eq. (11) into (10), we get the system:

$$
\frac{y_{i-1}-2 y_{i}+y_{i+1}}{h^{2}}=\alpha\left(M_{i-1}+M_{i+1}\right)+2 \beta M_{i} \text {, }
$$

where, $i=1,2, \ldots, N-1$,

$\alpha=\frac{\theta^{2}-2(1-\cos \theta)}{2 \theta^{2}(1-\cos \theta)} \quad$ and $\beta=\frac{4(1-\cos \theta)+\theta^{2}(1-3 \cos \theta)}{4 \theta^{2}(1-\cos \theta)}$.

If $h \rightarrow 0$, then $\theta=h k \rightarrow 0$. Thus using L'Hospital's rule we have $\lim _{\theta \rightarrow 0} \alpha=\frac{1}{12}$ and $\lim _{\theta \rightarrow 0} \beta=\frac{5}{12}$.

Using the splines second derivatives in Eqs. (9) with (4), we have

$$
\begin{aligned}
& M_{i}=\frac{1}{\varepsilon}\left(f_{i}-\mu p_{i} y_{i}^{\prime}+q_{i} y_{i}\right), \\
& M_{i-1}=\frac{1}{\varepsilon}\left(f_{i-1}-\mu p_{i-1} y_{i-1}^{\prime}+q_{i-1} y_{i-1}\right), \\
& M_{i+1}=\frac{1}{\varepsilon}\left(f_{i+1}-\mu p_{i+1} y_{i+1}^{\prime}+q_{i+1} y_{i+1}\right) .
\end{aligned}
$$

For the first-order derivatives in Eq. (13), we consider the central finite difference approximation of the form: 


$$
\begin{gathered}
y_{i}^{\prime}=\frac{y_{i+1}-y_{i-1}}{2 h}, \quad y_{i-1}^{\prime}=\frac{-3 y_{i-1}+4 y_{i}-y_{i+1}}{2 h}, \\
y_{i+1}^{\prime}=\frac{y_{i-1}-4 y_{i}+3 y_{i+1}}{2 h} .
\end{gathered}
$$

Using this approximation and substituting Eq. (13) into (12) yields:

$\left(\frac{\varepsilon}{h^{2}}+\frac{\mu}{2 h}\left(\alpha p_{i+1}-2 \beta p_{i}-3 \alpha p_{i-1}\right)-\alpha q_{i-1}\right) y_{i-1}$

$-\left(\frac{2 \varepsilon}{h^{2}}+\frac{2 \mu}{h}\left(\alpha p_{i+1}-\alpha p_{i-1}\right)+2 \beta q_{i}\right) y_{i}$

$+\left(\frac{\varepsilon}{h^{2}}+\frac{\mu}{2 h}\left(3 \alpha p_{i+1}+2 \beta p_{i}-\alpha p_{i-1}\right)-\alpha q_{i+1}\right) y_{i+1}=\alpha\left(f_{i+1}+f_{i-1}\right)+2 \beta f_{i}$.

To control the disturbance perturbation parameter in the solution, we introduce the fitting parameter $\sigma$ on Eq. (14). In order to get the value of $\sigma$, multiply Eq. (14) by $\frac{h}{\mu}$, denote $\rho=\frac{\mu h}{\varepsilon}$, and then evaluate limits as $h \rightarrow 0$ gives:

$$
\sigma=\frac{(\alpha+\beta) \rho p_{1} \lim _{h \rightarrow 0}\left(y_{i-1}-y_{i+1}\right)}{\lim _{h \rightarrow 0}\left(y_{i-1}-2 y_{i}+y_{i+1}\right)}
$$

From the discrete form of Eq. (7) we have

$$
\begin{aligned}
& y_{i}=C_{1}+C_{2} e^{\frac{-\mu p_{1}}{\varepsilon} x_{i}}=C_{1}+C_{2} e^{-p_{1} i \rho} \\
& y_{i+1}=C_{1}+C_{2} e^{-p_{1}(i+1) \rho}=C_{1}+C_{2} e^{-p_{1} i \rho} \cdot e^{-p_{1} \rho} \\
& y_{i-1}=C_{1}+C_{2} e^{-p_{1}(i-1) \rho}=C_{1}+C_{2} e^{-p_{1} i \rho} \cdot e^{p_{1} \rho}
\end{aligned}
$$

Considering Eq. (16) into Eq. (15), we get:

$$
\sigma=\frac{\rho p_{1}}{2} \operatorname{coth}\left(\frac{\rho p_{1}}{2}\right)
$$

Hence, the fitted form of Eq. (14) is

$$
E_{i} y_{i-1}-F_{i} y_{i}+G_{i} y_{i+1}=H_{i} \text {, }
$$

where

$$
\begin{aligned}
& E_{i}=\frac{\varepsilon \sigma}{h^{2}}+\frac{\mu}{2 h}\left(\alpha p_{i+1}-2 \beta p_{i}-3 \alpha p_{i-1}\right)-\alpha q_{i-1}, \\
& F_{i}=\frac{2 \varepsilon \sigma}{h^{2}}+\frac{2 \mu}{h}\left(\alpha p_{i+1}-\alpha p_{i-1}\right)+2 \beta q_{i}, \\
& G_{i}=\frac{\varepsilon \sigma}{h^{2}}+\frac{\mu}{2 h}\left(3 \alpha p_{i+1}+2 \beta p_{i}-\alpha p_{i-1}\right)-\alpha q_{i+1}, \\
& H_{i}=\alpha\left(f_{i+1}+f_{i-1}\right)+2 \beta f_{i} .
\end{aligned}
$$

\section{Error Analysis}

Let expand the terms $y_{i \pm 1}$ and $M_{i \pm 1}$ in Eq. (12), using Taylor's series which gives the local truncation error $T_{i}(h)$ :

$$
T_{i}(h)=(1-2(\alpha+\beta)) y_{i}^{\prime \prime}+h^{2}\left(\frac{1}{12}-\alpha\right) y_{i}^{(4)}+h^{4}\left(\frac{1}{360}-\frac{\alpha}{12}\right) y_{i}^{(6)}+\ldots
$$

But from the values of $\alpha=\frac{1}{12}$ and $\beta=\frac{5}{12}$, Eq. (18) leads to

$$
\left\|T_{i}(h)\right\| \leq C h^{4}
$$$$
\text { where, } C=\frac{1}{240}\left|y_{i}^{(6)}\right| \text {. }
$$

Thus, we have

$$
\left|y\left(x_{i}\right)-Y_{N}\right| \leq C\left(h^{4}\right)
$$

where $y\left(x_{i}\right)$ and $Y_{N}$ are exact and approximate solutions respectively, and $C$ is constant independent $h$.

To apply the Richardson extrapolation technique, let $\Omega^{2 N}$ obtained from each mesh interval $\Omega^{N}$ by dividing two, then denote the approximation of the solution on $\Omega^{2 N}$ by $Y_{2 N}$. Consider Eq. (20) works for any $h \neq 0$, which implies:

$$
y\left(x_{i}\right)-Y_{N} \cong C\left(h^{4}\right)+R^{N}, x_{i} \in \Omega^{N}
$$

So that it also works for any $\frac{h}{2} \neq 0$ and results:

$$
y\left(x_{i}\right)-Y_{2 N} \cong C\left(\left(\frac{h}{2}\right)^{4}\right)+R^{2 N}, x_{i} \in \Omega^{2 N}
$$

where the terms, $R^{N}$ and $R^{2 N}$ are of $O\left(h^{6}\right)$. Eliminating the constant $C$, and a combination of Eqs. (21) and (22) leads to $15 y\left(x_{i}\right)-\left(16 Y_{2 N}-Y_{N}\right) \approx O\left(h^{6}\right)$, which proposes to denote:

$$
\left(Y_{N}\right)^{e x t}=\frac{1}{15}\left(16 Y_{2 N}-Y_{N}\right)
$$

is also another approximation solution of $y\left(x_{i}\right)$ which obtained from the solutions of $Y_{N}$ ad $Y_{2 N}$. This approximation solution with the truncation error,

$$
\left|y\left(x_{i}\right)-\left(Y_{N}\right)^{e x t}\right| \leq C\left(h^{6}\right)
$$

Thus, the formulated quartic non-polynomial spline method in Eqs. (17) and extended to Eq. (23) with local truncation error in Eqs. (19) and (24) respectively, satisfies the consistency of the method if:

$$
\lim _{h \rightarrow 0} T_{i}(h)=\lim _{h \rightarrow 0} C h^{4}=\lim _{h \rightarrow 0} C h^{6}=0 .
$$

\section{Stability of the Method}

Let multiply both sides of the developed scheme in Eq. (17) by $h^{2}$ and consider the values of $E_{i}, F_{i}$ and $G_{i}$ for sufficiently small $h$, then we get:

$$
E_{i}=G_{i}=\varepsilon \sigma, F_{i}=2 \varepsilon \sigma
$$

Since, $i=1,2, \ldots, N-1$, considering Eq. (25), the matrix form of Eq. (17) is

$$
\begin{gathered}
A Y=B, \\
\text { where, }
\end{gathered}
$$




$$
\begin{aligned}
A & =\left[\begin{array}{cccccc}
-2 \varepsilon \sigma & \varepsilon \sigma & 0 & \ldots & \ldots & 0 \\
\varepsilon \sigma & -2 \varepsilon \sigma & \varepsilon \sigma & 0 & \ldots & \vdots \\
0 & \varepsilon \sigma & -2 \varepsilon \sigma & \varepsilon \sigma & \ddots & \vdots \\
\vdots & \ddots & \ddots & \ddots & \ddots & 0 \\
\vdots & \ddots & 0 & \varepsilon \sigma & -2 \varepsilon \sigma & \varepsilon \sigma \\
0 & \cdots & 0 & \varepsilon \sigma & -2 \varepsilon \sigma
\end{array}\right], \\
Y & =\left[\begin{array}{c}
0 \\
y_{1} \\
y_{2} \\
\vdots \\
\vdots \\
y_{N-2} \\
y_{N-1}
\end{array}\right] \text { and } B=\left[\begin{array}{c}
h^{2} H_{1}-E_{1} y_{0} \\
h^{2} H_{2} \\
\vdots \\
\vdots \\
h^{2} H_{N-2} \\
h^{2} H_{N-1}-G_{N-1} y_{N}
\end{array}\right] .
\end{aligned}
$$

Here, the matrix $A$ is a tri-diagonal, irreducible, and diagonally dominant. Therefore, the system can be solved by Thomas Algorithm.

As discussed in the literature $[16,18]$ the eigenvalues of a tri-diagonal matrix $A$ are given by:

$$
\lambda_{s}=-2 \varepsilon \sigma+2\{\sqrt{(\varepsilon \sigma)(\varepsilon \sigma)}\} \cos \frac{s \pi}{N}, \quad s=1(1) N-1 .
$$

Hence, the eigenvalues of the matrix $A$ in Eq. (26) are: $\lambda_{s}=-2 \varepsilon \sigma+2 \sqrt{(\varepsilon \sigma)^{2}} \cos \frac{s \pi}{N}=-2 \varepsilon \sigma\left(1-\cos \frac{s \pi}{N}\right), s=1(1) N-1$.

But from trigonometric identity, we have $1-\cos \frac{s \pi}{N}=2 \sin ^{2} \frac{s \pi}{2 N}$. Thus, the eigenvalues of $A$

$$
\lambda_{s}=-2 \varepsilon \sigma\left(2 \sin ^{2} \frac{s \pi}{2 N}\right)=-4 \varepsilon \sigma \sin ^{2} \frac{s \pi}{2 N} \leq-4 \varepsilon \sigma .
$$

A developed method is stable if $A$ is non-singular and $\left\|A^{-1}\right\| \leq C \quad \forall 0<h<h_{0}$.

where, $C$ and $h_{0}$ are two constants that are free of $h$.
Since $A$ is real and symmetric it follows that $A^{-1}$ is also. So that, its eigenvalues are given by $\frac{1}{\lambda_{s}}$. The stability condition of the method will be satisfied when

$$
\left\|A^{-1}\right\|=\left|\frac{1}{\lambda_{s}}\right|=\left|\frac{-1}{4 \varepsilon \sigma}\right|=\frac{1}{4 \varepsilon \sigma} \leq C .
$$

Thus, the developed quartic non-polynomial spline method is consistent and stable. Therefore, the proposed method is convergent.

\section{Numerical Illustrations}

In this section, we consider model examples of the singularly perturbed differential-difference equations with two parameters to validate our theoretical descriptions. Maximum absolute errors are computed by the formula:

$$
Z_{h}=\max _{i}\left|y_{i}^{h}-y_{i}^{\frac{h}{2}}\right| \quad i=1(1) N-1,
$$

where $y_{i}^{h}$ is the numerical solution at the nodal point $x_{i}$ on the mesh interval of $\Omega^{N}$ and $y_{i}^{\frac{h}{2}}$ is the numerical solution at the nodal point $x_{i}$ on the mesh interval of $\Omega^{2 N}$.

Example 1: Consider the singularly perturbed differentialdifference problem

$$
\left\{\begin{array}{l}
\varepsilon y^{\prime \prime}(x)+\mu(1+x) y^{\prime}(x-\delta)-e^{-x} y(x)=0, \quad x \in(0,1) \\
y(x)=1,-\delta \leq x \leq 0, \quad y(1)=1 .
\end{array}\right.
$$

The exact solution is not available, so we calculate the maximum absolute errors by the double mesh principle. For computational purposes, we consider $\delta=10^{-12}$ for both examples.

Example 2: Consider the singularly perturbed differential-

\begin{tabular}{|c|c|c|c|c|c|c|}
\hline$\mu \downarrow$ & $N \rightarrow 32$ & 64 & 128 & 256 & 512 & 1024 \\
\hline \multicolumn{7}{|c|}{ Present Method } \\
\hline $10^{-2}$ & $6.5542 \mathrm{e}-05$ & $1.6398 \mathrm{e}-05$ & $4.0996 \mathrm{e}-06$ & $1.0249 \mathrm{e}-06$ & $2.5634 \mathrm{e}-07$ & $6.4086 \mathrm{e}-08$ \\
\hline $10^{-4}$ & $7.9774 \mathrm{e}-06$ & $5.2550 \mathrm{e}-06$ & $1.4139 \mathrm{e}-06$ & $3.6245 \mathrm{e}-07$ & $9.0642 \mathrm{e}-08$ & $2.2661 \mathrm{e}-08$ \\
\hline $10^{-8}$ & $1.4789 \mathrm{e}-05$ & $4.2908 \mathrm{e}-07$ & $7.5055 \mathrm{e}-09$ & $8.8815 \mathrm{e}-11$ & $7.2546 \mathrm{e}-12$ & $2.0887 \mathrm{e}-12$ \\
\hline $10^{-10}$ & $1.4791 \mathrm{e}-05$ & $4.2964 \mathrm{e}-07$ & $7.6475 \mathrm{e}-09$ & $1.2503 \mathrm{e}-10$ & $1.9034 \mathrm{e}-12$ & $7.1987 \mathrm{e}-13$ \\
\hline \multicolumn{7}{|c|}{ Results in [9] } \\
\hline $10^{-2}$ & $7.074 \mathrm{e}-03$ & $1.945 \mathrm{e}-03$ & $5.018 \mathrm{e}-04$ & $1.271 \mathrm{e}-04$ & $3.184 \mathrm{e}-05$ & $7.968 \mathrm{e}-06$ \\
\hline $10^{-4}$ & $1.045 \mathrm{e}-02$ & $2.830 \mathrm{e}-03$ & $7.229 \mathrm{e}-04$ & $1.817 \mathrm{e}-04$ & $4.549 \mathrm{e}-05$ & $1.137 \mathrm{e}-05$ \\
\hline $10^{-8}$ & $1.047 \mathrm{e}-02$ & $2.835 \mathrm{e}-03$ & $7.242 \mathrm{e}-04$ & $1.820 \mathrm{e}-04$ & $4.557 \mathrm{e}-05$ & $1.139 \mathrm{e}-05$ \\
\hline $10^{-10}$ & $1.047 \mathrm{e}-02$ & $2.835 \mathrm{e}-03$ & $7.242 \mathrm{e}-04$ & $1.820 \mathrm{e}-04$ & $4.557 \mathrm{e}-05$ & $1.139 \mathrm{e}-05$ \\
\hline
\end{tabular}
difference problem

$$
\left\{\begin{array}{l}
\varepsilon y^{\prime \prime}(x)+\mu y^{\prime}(x-\delta)-y(x)=-x, \quad x \in(0,1) \\
y(x)=1, \quad-\delta \leq x \leq 0, \quad y(1)=0 .
\end{array}\right.
$$

The analytic solution of this problem is given by:

$y(x)=\frac{(1-\mu)\left(e^{-\left(k_{1}+k_{2} x\right) /\left(2 \varepsilon_{1}\right)}-e^{-\left(k_{1} x+k_{2}\right) /\left(2 \varepsilon_{1}\right)}\right)+(1+\mu)\left(e^{-k_{2} x /\left(2 \varepsilon_{1}\right)}-e^{-k_{1} x /\left(2 \varepsilon_{1}\right)}\right)}{\left(e^{-k_{1} /\left(2 \varepsilon_{1}\right)}-e^{-k_{2} /\left(2 \varepsilon_{1}\right)}\right)}+(\mu+x)$

$$
\text { with } \varepsilon_{1}=(\varepsilon-\mu \delta) \text { and } k_{1,2}=\mu \pm \sqrt{\mu^{2}+4 \varepsilon_{1}}
$$

Table 1 Comparison of maximum absolute errors for Example 1, when $\varepsilon=10^{-3}$ 
G. R. Kusi, T. A. Bullo, and G. F. Duressa /JEA Vol. 02(02) 2021, pp 71-77

Table 2 Comparison of maximum absolute errors for Example 1, when $\mu=10^{-4}$

\begin{tabular}{lllllll}
\hline$\varepsilon \downarrow$ & $N \rightarrow 32$ & 64 & 128 & 256 & 512 & 1024 \\
\hline \multicolumn{2}{l}{ Present Method } & & & & \\
$10^{-2}$ & $6.9549 \mathrm{e}-07$ & $1.8398 \mathrm{e}-07$ & $4.6162 \mathrm{e}-08$ & $1.1543 \mathrm{e}-08$ & $2.8867 \mathrm{e}-09$ & $7.2070 \mathrm{e}-10$ \\
$10^{-4}$ & $7.4475 \mathrm{e}-04$ & $4.0798 \mathrm{e}-05$ & $2.7815 \mathrm{e}-05$ & $7.5115 \mathrm{e}-06$ & $1.8890 \mathrm{e}-06$ & $4.7538 \mathrm{e}-07$ \\
$10^{-8}$ & $1.3837 \mathrm{e}-03$ & $5.9743 \mathrm{e}-05$ & $3.9377 \mathrm{e}-05$ & $1.0955 \mathrm{e}-05$ & $2.8532 \mathrm{e}-06$ & $7.1372 \mathrm{e}-07$ \\
$10^{-10}$ & $1.3837 \mathrm{e}-03$ & $5.9743 \mathrm{e}-05$ & $3.9377 \mathrm{e}-05$ & $1.0955 \mathrm{e}-05$ & $2.8532 \mathrm{e}-06$ & $7.1372 \mathrm{e}-07$ \\
Results in [9] & & & & & \\
$10^{-2}$ & $1.252 \mathrm{e}-03$ & $3.162 \mathrm{e}-04$ & $7.946 \mathrm{e}-05$ & $1.987 \mathrm{e}-05$ & $4.970 \mathrm{e}-06$ & $1.242 \mathrm{e}-06$ \\
$10^{-4}$ & $2.470 \mathrm{e}-02$ & $1.301 \mathrm{e}-02$ & $4.656 \mathrm{e}-03$ & $1.624 \mathrm{e}-03$ & $4.396 \mathrm{e}-04$ & $1.102 \mathrm{e}-04$ \\
$10^{-8}$ & $2.123 \mathrm{e}-02$ & $8.699 \mathrm{e}-03$ & $3.120 \mathrm{e}-03$ & $1.052 \mathrm{e}-03$ & $3.347 \mathrm{e}-04$ & $1.038 \mathrm{e}-04$ \\
$10^{-10}$ & $2.121 \mathrm{e}-02$ & $7.835 \mathrm{e}-03$ & $2.704 \mathrm{e}-03$ & $8.976 \mathrm{e}-04$ & $2.797 \mathrm{e}-04$ & $8.430 \mathrm{e}-04$ \\
\hline
\end{tabular}

Table 3 Maximum absolute errors before and after applying the Richardson extrapolation for Example 1 , when $\varepsilon<\mu=10^{-3}$

\begin{tabular}{lllllll}
\hline$\varepsilon \downarrow$ & $N \rightarrow 32$ & 64 & 128 & 256 & 512 & 1024 \\
\hline After & & & & & & \\
$10^{-4}$ & $1.8079 \mathrm{e}-04$ & $5.4565 \mathrm{e}-05$ & $1.3834 \mathrm{e}-05$ & $3.5207 \mathrm{e}-06$ & $8.8021 \mathrm{e}-07$ & $2.2005 \mathrm{e}-07$ \\
$10^{-6}$ & $1.8079 \mathrm{e}-04$ & $5.4565 \mathrm{e}-05$ & $1.3834 \mathrm{e}-05$ & $3.5207 \mathrm{e}-06$ & $8.8021 \mathrm{e}-07$ & $2.2005 \mathrm{e}-07$ \\
$10^{-8}$ & $1.8079 \mathrm{e}-04$ & $5.4565 \mathrm{e}-05$ & $1.3834 \mathrm{e}-05$ & $3.5207 \mathrm{e}-06$ & $8.8021 \mathrm{e}-07$ & $2.2005 \mathrm{e}-07$ \\
$10^{-10}$ & $1.8080 \mathrm{e}-04$ & $5.4567 \mathrm{e}-05$ & $1.3834 \mathrm{e}-05$ & $3.5208 \mathrm{e}-06$ & $8.8024 \mathrm{e}-07$ & $2.2006 \mathrm{e}-07$ \\
Before & & & & & \\
$10^{-4}$ & $3.2137 \mathrm{e}-03$ & $1.3165 \mathrm{e}-04$ & $4.9202 \mathrm{e}-05$ & $1.6322 \mathrm{e}-05$ & $4.3208 \mathrm{e}-06$ & $1.0952 \mathrm{e}-06$ \\
$10^{-6}$ & $3.2137 \mathrm{e}-03$ & $1.3165 \mathrm{e}-04$ & $4.9202 \mathrm{e}-05$ & $1.6322 \mathrm{e}-05$ & $4.3208 \mathrm{e}-06$ & $1.0952 \mathrm{e}-06$ \\
$10^{-8}$ & $3.2137 \mathrm{e}-03$ & $1.3165 \mathrm{e}-04$ & $4.9202 \mathrm{e}-05$ & $1.6322 \mathrm{e}-05$ & $4.3208 \mathrm{e}-06$ & $1.0952 \mathrm{e}-06$ \\
$10^{-10}$ & $3.2138 \mathrm{e}-03$ & $1.3165 \mathrm{e}-04$ & $4.9203 \mathrm{e}-05$ & $1.6322 \mathrm{e}-05$ & $4.3209 \mathrm{e}-06$ & $1.0953 \mathrm{e}-06$ \\
\hline
\end{tabular}

Table 4 Maximum absolute errors with fitting (W. F) and without fitting (W.O. F) parameter for Example 1 , when $\varepsilon<\mu=10^{-3}$

\begin{tabular}{lllllll}
\hline$\varepsilon \downarrow$ & $N \rightarrow 32$ & 64 & 128 & 256 & 512 & 1024 \\
\hline W. F & $1.8079 \mathrm{e}-04$ & $5.4565 \mathrm{e}-05$ & $1.3834 \mathrm{e}-05$ & $3.5207 \mathrm{e}-06$ & $8.8021 \mathrm{e}-07$ & $2.2005 \mathrm{e}-07$ \\
$10^{-4}$ & $1.8079 \mathrm{e}-04$ & $5.4565 \mathrm{e}-05$ & $1.3834 \mathrm{e}-05$ & $3.5207 \mathrm{e}-06$ & $8.8021 \mathrm{e}-07$ & $2.2005 \mathrm{e}-07$ \\
$10^{-6}$ & $1.8079 \mathrm{e}-04$ & $5.4565 \mathrm{e}-05$ & $1.3834 \mathrm{e}-05$ & $3.5207 \mathrm{e}-06$ & $8.8021 \mathrm{e}-07$ & $2.2005 \mathrm{e}-07$ \\
$10^{-8}$ & $1.8080 \mathrm{e}-04$ & $5.4567 \mathrm{e}-05$ & $1.3834 \mathrm{e}-05$ & $3.5208 \mathrm{e}-06$ & $8.8024 \mathrm{e}-07$ & $2.2006 \mathrm{e}-07$ \\
$10^{-10}$ & & & & & \\
W.O. F & $1.3710 \mathrm{e}-03$ & $8.5454 \mathrm{e}-04$ & $3.9479 \mathrm{e}-04$ & $1.0020 \mathrm{e}-04$ & $2.5467 \mathrm{e}-05$ & $6.3837 \mathrm{e}-06$ \\
$10^{-4}$ & $2.6758 \mathrm{e}-02$ & $3.3937 \mathrm{e}-02$ & $3.5052 \mathrm{e}-02$ & $1.2619 \mathrm{e}-02$ & $5.9584 \mathrm{e}-03$ & $4.4740 \mathrm{e}-03$ \\
$10^{-6}$ & $2.8268 \mathrm{e}-02$ & $4.0314 \mathrm{e}-02$ & $6.1232 \mathrm{e}-02$ & $8.5222 \mathrm{e}-02$ & $1.3035 \mathrm{e}-01$ & $2.5806 \mathrm{e}-01$ \\
$10^{-8}$ & $2.8283 \mathrm{e}-02$ & $4.0349 \mathrm{e}-02$ & $6.0781 \mathrm{e}-02$ & $7.6575 \mathrm{e}-02$ & $7.4437 \mathrm{e}-02$ & $8.2861 \mathrm{e}-02$ \\
$10^{-10}$ & & & & &
\end{tabular}

Table 5 Comparison of maximum absolute errors for Example 2, when $\varepsilon=10^{-3}$

\begin{tabular}{lllllll}
\hline$\mu \downarrow$ & $N \rightarrow 32$ & 64 & 128 & 256 & 512 & 1024 \\
\hline \multicolumn{2}{l}{ Present Method } & & & & \\
$10^{-2}$ & $9.2960 \mathrm{e}-04$ & $2.3290 \mathrm{e}-04$ & $5.8941 \mathrm{e}-05$ & $1.4738 \mathrm{e}-05$ & $3.6893 \mathrm{e}-06$ & $3.6893 \mathrm{e}-06$ \\
$10^{-5}$ & $2.0555 \mathrm{e}-06$ & $2.5750 \mathrm{e}-07$ & $5.9506 \mathrm{e}-08$ & $1.4797 \mathrm{e}-08$ & $3.6979 \mathrm{e}-09$ & $9.2472 \mathrm{e}-10$ \\
$10^{-7}$ & $1.1198 \mathrm{e}-06$ & $2.3221 \mathrm{e}-08$ & $9.3234 \mathrm{e}-10$ & $1.5330 \mathrm{e}-10$ & $3.7066 \mathrm{e}-11$ & $9.4675 \mathrm{e}-12$ \\
$10^{-10}$ & $1.1103 \mathrm{e}-06$ & $2.0857 \mathrm{e}-08$ & $3.4127 \mathrm{e}-10$ & $5.5301 \mathrm{e}-12$ & $1.4166 \mathrm{e}-13$ & $3.9091 \mathrm{e}-13$ \\
Results in $[9]$ & & & & & \\
$10^{-2}$ & $1.470 \mathrm{e}-02$ & $3.909 \mathrm{e}-03$ & $1.005 \mathrm{e}-03$ & $2.525 \mathrm{e}-04$ & $6.327 \mathrm{e}-05$ & $1.582 \mathrm{e}-05$ \\
$10^{-5}$ & $1.071 \mathrm{e}-02$ & $3.666 \mathrm{e}-03$ & $9.318 \mathrm{e}-04$ & $2.339 \mathrm{e}-04$ & $5.854 \mathrm{e}-05$ & $1.464 \mathrm{e}-05$ \\
$10^{-7}$ & $1.066 \mathrm{e}-02$ & $3.660 \mathrm{e}-03$ & $9.303 \mathrm{e}-04$ & $2.335 \mathrm{e}-04$ & $5.844 \mathrm{e}-05$ & $1.461 \mathrm{e}-05$ \\
$10^{-10}$ & $1.066 \mathrm{e}-02$ & $3.660 \mathrm{e}-03$ & $9.303 \mathrm{e}-04$ & $2.335 \mathrm{e}-04$ & $5.844 \mathrm{e}-05$ & $1.461 \mathrm{e}-05$ \\
\hline
\end{tabular}


Table 6 Comparison of maximum absolute errors for Example 2, when $\varepsilon=10^{-3}$.

\begin{tabular}{llllllr}
\hline$\mu \downarrow$ & $N \rightarrow 32$ & 64 & 128 & 256 & 512 & 1024 \\
\hline After & & & & & & \\
$10^{-4}$ & $1.0592 \mathrm{e}-05$ & $2.3946 \mathrm{e}-06$ & $5.9382 \mathrm{e}-07$ & $1.4838 \mathrm{e}-07$ & $3.7093 \mathrm{e}-08$ & $9.2732 \mathrm{e}-09$ \\
$10^{-6}$ & $1.2048 \mathrm{e}-06$ & $4.4520 \mathrm{e}-08$ & $6.2575 \mathrm{e}-09$ & $1.4846 \mathrm{e}-09$ & $3.6988 \mathrm{e}-10$ & $9.2715 \mathrm{e}-11$ \\
$10^{-8}$ & $1.1113 \mathrm{e}-06$ & $2.1091 \mathrm{e}-08$ & $3.9984 \mathrm{e}-10$ & $2.0174 \mathrm{e}-11$ & $3.7818 \mathrm{e}-12$ & $1.1909 \mathrm{e}-12$ \\
$10^{-10}$ & $1.1103 \mathrm{e}-06$ & $2.0857 \mathrm{e}-08$ & $3.4127 \mathrm{e}-10$ & $5.5301 \mathrm{e}-12$ & $1.4166 \mathrm{e}-13$ & $3.9091 \mathrm{e}-13$ \\
Before & & & & & \\
$10^{-4}$ & $7.5786 \mathrm{e}-04$ & $5.7296 \mathrm{e}-05$ & $5.8259 \mathrm{e}-06$ & $9.2083 \mathrm{e}-07$ & $1.9666 \mathrm{e}-07$ & $4.7066 \mathrm{e}-08$ \\
$10^{-6}$ & $7.0795 \mathrm{e}-04$ & $4.5377 \mathrm{e}-05$ & $2.8778 \mathrm{e}-06$ & $1.8573 \mathrm{e}-07$ & $1.3000 \mathrm{e}-08$ & $1.1592 \mathrm{e}-09$ \\
$10^{-8}$ & $7.0746 \mathrm{e}-04$ & $4.5258 \mathrm{e}-05$ & $2.8484 \mathrm{e}-06$ & $1.7840 \mathrm{e}-07$ & $1.1169 \mathrm{e}-08$ & $7.0160 \mathrm{e}-10$ \\
$10^{-10}$ & $7.0745 \mathrm{e}-04$ & $4.5257 \mathrm{e}-05$ & $2.8481 \mathrm{e}-06$ & $1.7833 \mathrm{e}-07$ & $1.1151 \mathrm{e}-08$ & $6.9702 \mathrm{e}-10$ \\
\hline
\end{tabular}

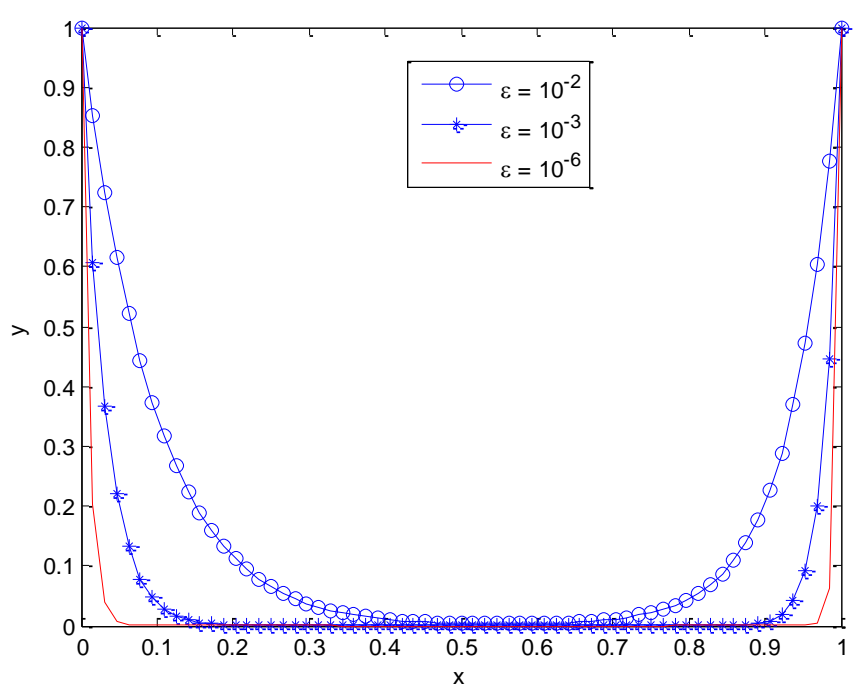

Fig. 1 Solution profiles for Example 1, when $\mu=10^{-4}$,

$$
\delta=10^{-12} \text { and } N=64 \text {. }
$$

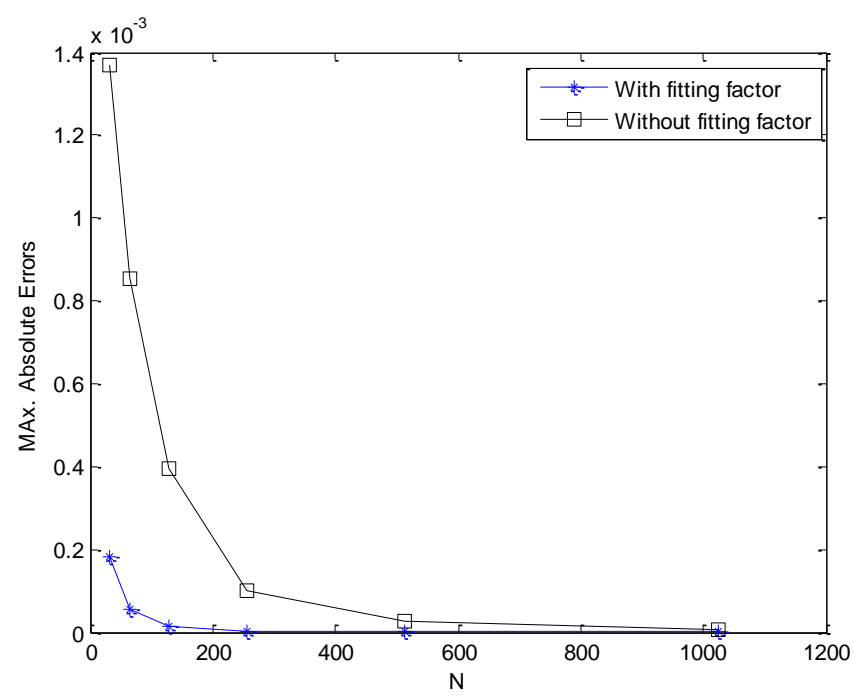

Fig. 2 Obtained maximum absolute errors when $\varepsilon=10^{-4}$ and

$$
\mu=10^{-3}
$$

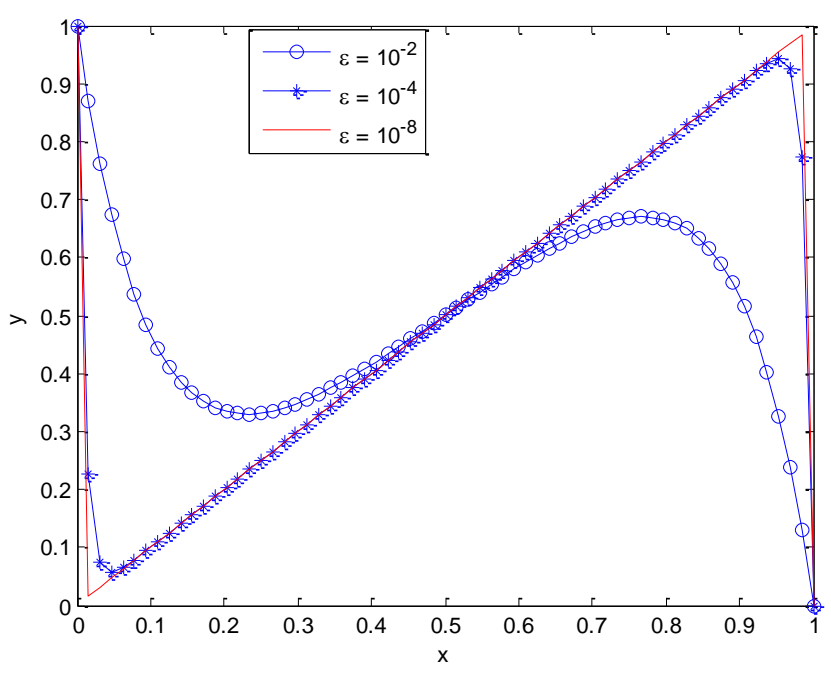

Fig. 3 Solution profiles for Example 2, when $\mu=10^{-4}$,

$$
\delta=10^{-12} \text { and } N=64 \text {. }
$$

\section{Discussion and Conclusion}

In this paper, we presented a quartic non-polynomial spline method to solve the singularly perturbed differential-difference equation involving two parameters. First, this equation is transformed into an asymptotically equivalent differential equation, and then applying a quartic non-polynomial spline method. Convergence of the method has been established. To validate the method, numerical illustrations have been given in Table 1-6 and Fig. 1-3.

These results show, the present method gives a more accurate solution than some existing methods in the literature. Also, maximum absolute errors decrease as the number of mesh points $N$ increases which implies convergence of the method. Moreover, we attempted to increase the order of convergence by Richardson's extrapolation and discovered that this well-known convergence acceleration technique has some limitations. We observe that even though this extrapolation technique improves the accuracy slightly, it does not increase the rate of convergence.

Generally, a quartic non-polynomial spline method is convergent and gives an accurate numerical solution for solving singularly perturbed differential-difference problems involving two parameters. 


\section{References}

[1] File, G. and Reddy, Y.N., 2013. Computational method for solving singularly perturbed delay differential equations with negative shift. International Journal of Applied Science and Engineering, 11(1), pp.101-113.

[2] File, G., Gadisa, G., Aga, T. and Reddy, Y.N., 2017. Numerical solution of singularly perturbed delay reactiondiffusion equations with layer or oscillatory behaviour. American Journal of Numerical Analysis, 5(1), pp.1-10.

[3] Gadisa, G., File, G. and Aga, T., 2018. Fourth order numerical method for singularly perturbed delay differential equations. International Journal of Applied Science and Engineering, 15(1), pp.17-32.

[4] Kadalbajoo, M.K. and Kumar, V., 2007. B-spline method for a class of singular two-point boundary value problems using optimal grid. Applied Mathematics and Computation, 188(2), pp.1856-1869.

[5] Kadalbajoo, M.K. and Sharma, K.K., 2004. Numerical analysis of singularly perturbed delay differential equations with layer behavior. Applied Mathematics and Computation, 157(1), pp.11-28.

[6] Kadalbajoo, M.K. and Sharma, K.K., 2008. A numerical method based on finite difference for boundary value problems for singularly perturbed delay differential equations. Applied Mathematics and Computation, 197(2), pp.692-707.

[7] Kadalbajoo, M.K. and Yadaw, A.S., 2008. B-Spline collocation method for a two-parameter singularly perturbed convection-diffusion boundary value problems. Applied Mathematics and Computation, 201(12), pp.504-513.

[8] Kadalbajoo, M.K., Gupta, V. and Awasthi, A., 2008. A uniformly convergent B-spline collocation method on a nonuniform mesh for singularly perturbed onedimensional time-dependent linear convection-diffusion problem. Journal of Computational and Applied Mathematics, 220(1-2), pp.271-289.

[9] Sahu, S.R. and Mohapatra, J., 2019. Parameter uniform numerical methods for singularly perturbed delay differential equation involving two small parameters. International Journal of Applied and Computational Mathematics, 5(5), pp.1-19.

[10] Akram, G. and Talib, I., 2014. Quartic non-polynomial spline solution of a third order singularly perturbed boundary value problem. Research Journal of Applied Sciences, Engineering and Technology, 7(23), pp.48594863.

[11] Ala'yed, O.H., Ying, T.Y. and Saaban, A., 2015. New fourth order quartic spline method for solving second order boundary value problems. MATEMATIKA: Malaysian Journal of Industrial and Applied Mathematics, 31(2), pp.149-157.

[12] Chakravarthy, P.P., Kumar, S.D., Rao, R.N. and Ghate, D.P., 2015. A fitted numerical scheme for second order singularly perturbed delay differential equations via cubic spline in compression. Advances in Difference Equations, 2015(1), pp.1-14.

[13] Chakravarthy, P.P., Kumar, S.D. and Rao, R.N., 2017. An exponentially fitted finite difference scheme for a class of singularly perturbed delay differential equations with large delays. Ain Shams Engineering Journal, 8(4), pp.663-671.

[14] Dugassa, T., File, G. and Aga, T., 2019. Stable Numerical Method for Singularly Perturbed Boundary Value Problems with Two Small Parameters. Ethiopian Journal of Education and Sciences, 14(2), pp.9-27.

[15] Erdogan, F., 2009. An exponentially fitted method for singularly perturbed delay differential equations. Advances in Difference Equations, 2009, pp.19.

[16] Siraj, M.K., Duressa, G.F. and Bullo, T.A., 2019. Fourthorder stable central difference with Richardson extrapolation method for second-order self-adjoint singularly perturbed boundary value problems. Journal of the Egyptian Mathematical Society, 27(1), pp.1-14.

[17] Zahra, W.K. and El Mhlawy, A.M., 2013. Numerical solution of two-parameter singularly perturbed boundary value problems via exponential spline. Journal of King Saud University-Science, 25(3), pp.201-208.

[18] Smith, G.D., Smith, G.D. and Smith, G.D.S., 1985. Numerical solution of partial differential equations: finite difference methods. Oxford University Press. 